PROCEEDINGS OF THE

AMERICAN MATHEMATICAL SOCIETY

Volume 131, Number 8, Pages 2561-2571

S 0002-9939(03)07082-5

Article electronically published on March 18, 2003

\title{
RECONSTRUCTION OF FUNCTIONS IN SPLINE SUBSPACES FROM LOCAL AVERAGES
}

\author{
WENCHANG SUN AND XINGWEI ZHOU
}

(Communicated by David R. Larson)

\begin{abstract}
In this paper, we study the reconstruction of functions in spline subspaces from local averages. We present an average sampling theorem for shift invariant subspaces generated by cardinal B-splines and give the optimal upper bound for the support length of averaging functions. Our result generalizes an earlier result by Aldroubi and Gröchenig.
\end{abstract}

\section{Introduction AND the MAIN RESUlt}

The sampling theorem is one of the most powerful results in signal analysis. It states that if a signal (function) $f(x)$ satisfies certain conditions, then it is uniquely determined by its sampled values at some discrete points $\left\{x_{k}: k \in \mathbb{Z}\right\}$ and

$$
f(x)=\sum_{k \in \mathbb{Z}} f\left(x_{k}\right) S_{k}(x)
$$

for some sampling functions $\left\{S_{k}(x)\right\}$. For example, every $f \in B_{\Omega}:=\{f: \operatorname{supp} \hat{f} \subset$ $[-\Omega, \Omega]\}$ can be reconstructed by the formula $f(x)=\sum_{k \in \mathbb{Z}} f\left(\frac{k \pi}{\Omega}\right) \frac{\sin (\Omega x-k \pi)}{\Omega x-k \pi}$. This is the classical Shannon sampling theorem. Although the assumption that a signal is band-limited is eminently useful, it is not always realistic since a band-limited signal is of infinite duration. Thus, it is natural to investigate other signal classes for which a sampling theorem holds. A simple model is to consider shift-invariant subspaces, e.g., wavelet subspaces, which generalize the space of band-limited functions and are of the form

$$
V=\overline{\operatorname{span}}\{\varphi(\cdot-k): k \in \mathbb{Z}\}
$$

for some kernel function $\varphi(x)$. In fact, there have been many results concerning the sampling in shift-invariant subspaces for both regular and irregular sampling. For details, see [1]-[7], [10]-[19], [22]-[25] and [27].

Received by the editors May 11, 2001.

2000 Mathematics Subject Classification. Primary 94A20; Secondary 42C40, 42C15.

Key words and phrases. Average sampling, sampling theorems, spline subspaces.

This work was supported by the Research Fund for the Doctoral Program of Higher Education, the National Natural Science Foundation of China (Grant Nos. 10171050 and 10201014), the Mathematical Tianyuan Foundation(Grant No. TY10126007), and the Liuhui Center for Applied Mathematics. 
In particular, for the spline subspace

$$
V_{m}=\left\{\sum_{k \in \mathbb{Z}} c_{k} \varphi_{m}(\cdot-k):\left\{c_{k}\right\} \in \ell^{2}\right\}
$$

generated by the cardinal B-spline

$$
\varphi_{m}=\chi_{[0,1]} * \cdots * \chi_{[0,1]}(m+1 \text { terms }), \quad m \geq 1,
$$

Liu [16] proved that every $f \in V_{m}$ is uniquely determined and can be reconstructed by an iterative algorithm from its samples $f\left(x_{k}\right)$ if $x_{k+1}-x_{k}$ is small enough. Furthermore, Aldroubi and Gröchenig [1] proved that if $\alpha \leq x_{k+1}-x_{k} \leq \beta$ for some constants $0<\alpha<\beta<1$, then every $f \in V_{m}$ is uniquely determined by $f\left(x_{k}\right)$.

For physical reasons, e.g., the inertia of the measurement apparatus, measured sampled values obtained in practice may not be values of a function $f$ precisely at times $x_{k}$, but only local averages of $f$ near $x_{k}$. Specifically, measured sampled values are

$$
\left\langle f, u_{k}\right\rangle=\int f(x) u_{k}(x) d x
$$

for some collection of averaging functions $u_{k}(x), k \in \mathbb{Z}$, which satisfy the following properties:

$$
\text { supp } u_{k} \subset\left[x_{k}-\frac{\delta}{2}, x_{k}+\frac{\delta}{2}\right], \quad u_{k}(x) \geq 0, \quad \text { and } \quad \int u(x) d x=1 .
$$

Observe that the averaging procedure is allowed to vary from point to point.

It is clear that from local averages one should obtain at least a good approximation of the original signal if $\delta$ is small enough. Wiley, Butzer and Lei [5, 6, 24] studied the approximation error when local averages are used as sampled values. Furthermore, Gröchenig [14] proved that if sampling points $\left\{x_{k}: k \in \mathbb{Z}\right\}$ satisfy $0<x_{k+1}-x_{k} \leq \delta<\frac{1}{\sqrt{2} \Omega}$, then every $f \in B_{\Omega}$ is uniquely determined by local averages $\left\langle f, u_{k}\right\rangle$ around $x_{k}$ and can be reconstructed by an iteration scheme. In [13], Feichtinger and Gröchenig proved that if $\delta:=\sup _{k \in \mathbb{Z}}\left(x_{k+1}-x_{k}\right)<\frac{\pi}{\Omega}$, then every $f \in B_{\Omega}$ is uniquely determined by $\frac{1}{y_{k}-y_{k-1}} \int_{y_{k-1}}^{y_{k}} f(x) d x$ with $y_{k}=\frac{x_{k}+x_{k+1}}{2}$.

In [20, we gave an average sampling theorem for shift invariant subspaces with equally spaced sampling points and arbitrary averaging functions. For the case of band-limited signals, we gave an analogy of Kadec's 1/4-theorem. In [21], we studied average sampling theorems for spline subspaces with standard averaging functions $u_{k}=\chi_{\left[x_{k}-1 / 2, x_{k}+1 / 2\right]}$.

In this paper, we study the reconstruction of functions in spline subspaces from local averages with arbitrary averaging functions and irregular sampling points. We present an average sampling theorem and give the optimal upper bound for the support length of averaging functions. Our result generalizes Aldroubi and Gröchenig's irregular sampling theorem. In fact, we prove the following.

Theorem 1.1. Let $\left\{x_{k}: k \in \mathbb{Z}\right\}$ be a real sequence such that $\lim _{k \rightarrow \pm \infty} x_{k}= \pm \infty$ and

$$
0<x_{k+1}-x_{k} \leq \beta<1, \quad k \in \mathbb{Z}
$$

for some constant $\beta$. Then for any $0<\delta<1-\beta$ and averaging functions $u_{k}(x)$ with supp $u_{k} \subset\left[x_{k}-\frac{\delta}{2}, x_{k}+\frac{\delta}{2}\right]$, there is a frame $\left\{S_{k}(x): k \in \mathbb{Z}\right\}$ for $V_{m}$ such that 
for any $f \in V_{m}$,

$$
f(x)=\sum_{k \in \mathbb{Z}}\left(\frac{x_{k+1}-x_{k-1}}{2}\right)^{1 / 2}\left\langle f, u_{k}\right\rangle S_{k}(x),
$$

where the convergence is both in $L^{2}(\mathbb{R})$ and uniform on $\mathbb{R}$.

Furthermore, the conclusion fails if $\delta \geq 1-\beta$.

Notation and definition. The Fourier transform and the Zak transform of $f \in$ $L^{2}(\mathbb{R})$ is defined by $\hat{f}(\omega)=\int_{\mathbb{R}} f(x) e^{-i x \omega} d x$ and $Z f(x, \omega)=\sum_{k \in \mathbb{Z}} f(x+k) e^{-i k \omega}$ respectively.

We call $u(x)$ an averaging function if $u(x) \geq 0, u(x) \in L^{1}(\mathbb{R})$ and $\int_{\mathbb{R}} u(x) d x=1$.

\section{SOME BASIC FACTS IN FRAME THEORY}

In this section, we introduce some basic facts in frame theory.

A family of functions $\left\{\varphi_{k}: k \in \mathbb{Z}\right\}$ belonging to a separable Hilbert space $\mathcal{H}$ is said to be a frame if there exist positive constants $A$ and $B$ such that $A\|f\|^{2} \leq$ $\sum_{k \in \mathbb{Z}}\left|\left\langle f, \varphi_{k}\right\rangle\right|^{2} \leq B\|f\|^{2}$ for every $f \in \mathcal{H}$. The constants $A$ and $B$ are called the lower and upper frame bounds respectively.

A frame that ceases to be a frame when any one of its elements is removed is said to be an exact frame. It is well known that exact frames and Riesz bases are identical (see [26]).

Let $\left\{\varphi_{k}: k \in \mathbb{Z}\right\}$ be a frame for some Hilbert space $\mathcal{H}$ with bounds $A$ and $B$. Define the frame operator $T$ as follows: $T f=\sum_{n \in \mathbb{Z}}\left\langle f, \varphi_{n}\right\rangle \varphi_{n}, \forall f \in \mathcal{H}$. Then $T$ is bounded and invertible and $\left\{T^{-1} \varphi_{k}: k \in \mathbb{Z}\right\}$ is also a frame for $\mathcal{H}$ with bounds $\frac{1}{B}$ and $\frac{1}{A}$, called the dual frame of $\left\{\varphi_{k}: k \in \mathbb{Z}\right\}$. It can be shown that for any $f \in \mathcal{H}$,

$$
f=\sum_{n \in \mathbb{Z}}\left\langle f, T^{-1} \varphi_{n}\right\rangle \varphi_{n}=\sum_{n \in \mathbb{Z}}\left\langle f, \varphi_{n}\right\rangle T^{-1} \varphi_{n} .
$$

For the general theory on frames and Riesz bases, see [9, 26].

For frames in shift invariant subspaces, we have the following.

Lemma 2.1. Let $\{\varphi(\cdot-k): k \in \mathbb{Z}\}$ be a frame for some closed subspace $V$ of $L^{2}(\mathbb{R})$. Suppose that $\varphi$ is continuous and $\sum_{k \in \mathbb{Z}}|\varphi(x-k)|^{2} \leq L<+\infty$. Then for any frame $\left\{S_{k}: k \in \mathbb{Z}\right\}$ of $V_{0}, \sum_{k \in \mathbb{Z}}\left|S_{k}(x)\right|^{2}$ is bounded on $\mathbb{R}$.

Proof. Let $\{\tilde{\varphi}(\cdot-k): k \in \mathbb{Z}\}$ be the dual frame of $\{\varphi(\cdot-k): k \in \mathbb{Z}\}$. Then for any $f \in V, f(x)=\sum_{k \in \mathbb{Z}}\langle f, \tilde{\varphi}(\cdot-k)\rangle \varphi(x-k)$. Therefore,

$$
\|f\|_{\infty}^{2} \leq \sup _{x} \sum_{k \in \mathbb{Z}}|\langle f, \tilde{\varphi}(\cdot-k)\rangle|^{2} \sum_{k \in \mathbb{Z}}|\varphi(x-k)|^{2} \leq \frac{L}{A}\|f\|_{2}^{2}
$$

It follows that for any $x \in \mathbb{R}$,

$$
\sum_{k \in \mathbb{Z}}\left|S_{k}(x)\right|^{2}=\sup _{\|c\|_{2}=1}\left|\sum_{k \in \mathbb{Z}} c_{k} S_{k}(x)\right|^{2} \leq \sup _{\|c\|_{2}=1} \frac{L}{A}\left\|\sum_{k \in \mathbb{Z}} c_{k} S_{k}\right\|_{2}^{2} \leq \frac{L M}{A},
$$

where $M$ is the upper frame bound of $\left\{S_{k}(x): k \in \mathbb{Z}\right\}$. 
Lemma 2.2. Suppose that $\left\{u_{k}: k \in \mathbb{Z}\right\}$ is a sequence of compactly supported averaging functions and there exist positive constants $A$ and $B$ such that

$$
A\|f\|^{2} \leq \sum_{k \in \mathbb{Z}}\left|\left\langle f, u_{k}\right\rangle\right|^{2} \leq B\|f\|^{2}, \quad \forall f \in V_{m} .
$$

Then there is a frame $\left\{S_{k}: k \in \mathbb{Z}\right\}$ for $V_{m}$ such that $f(x)=\sum_{k \in \mathbb{Z}}\left\langle f, u_{k}\right\rangle S_{k}(x), \forall f \in$ $V_{m}$, where the convergence is both in $L^{2}(\mathbb{R})$ and uniform on $\mathbb{R}$.

Proof. By [8, Theorem 4.5], $\left\{\varphi_{m}(\cdot-n): n \in \mathbb{Z}\right\}$ is a Riesz basis for $V_{m}$. Let $\left\{\tilde{\varphi}_{m}(\cdot-n): n \in \mathbb{Z}\right\}$ be the dual Riesz basis. For any $k \in \mathbb{Z}$, let

$$
h_{k}=\sum_{n \in \mathbb{Z}}\left\langle u_{k}, \varphi_{m}(\cdot-n)\right\rangle \tilde{\varphi}_{m}(\cdot-n) .
$$

Since both $\varphi_{m}$ and $u_{k}$ are compactly supported, the above series contains only finite non-zero terms. Hence $h_{k} \in V_{m}$. For any $f \in V_{m}$, we have

$$
\begin{aligned}
\left\langle f, h_{k}\right\rangle & =\sum_{n \in \mathbb{Z}}\left\langle\varphi_{m}(\cdot-n), u_{k}\right\rangle \cdot\left\langle f, \tilde{\varphi}_{m}(\cdot-n)\right\rangle \\
& =\left\langle\sum_{n \in \mathbb{Z}}\left\langle f, \tilde{\varphi}_{m}(\cdot-n)\right\rangle \varphi_{m}(\cdot-n), u_{k}\right\rangle \\
& =\left\langle f, u_{k}\right\rangle .
\end{aligned}
$$

Now (2.1) shows that $\left\{h_{k}: k \in \mathbb{Z}\right\}$ is a frame for $V_{m}$. Let $\left\{S_{k}: k \in \mathbb{Z}\right\}$ be the dual frame. Then for any $f \in V_{m}, f(x)=\sum_{k \in \mathbb{Z}}\left\langle f, h_{k}\right\rangle S_{k}(x)=\sum_{k \in \mathbb{Z}}\left\langle f, u_{k}\right\rangle S_{k}(x)$. The uniform convergence follows by Lemma 2.1.

\section{Proof of Theorem 1.1}

First, we introduce a result by Aldroubi and Gröchenig. The following proposition is a special case of [1, Theorem 1].

Proposition 3.1. Let $\left\{x_{k}: k \in \mathbb{Z}\right\}$ be a real sequence such that

$$
0<\alpha<x_{k+1}-x_{k}<\beta<1, \quad \forall k \in \mathbb{Z},
$$

for some constants $\alpha$ and $\beta$. Then there exist positive constants $C_{1}$ and $C_{2}$ depending only on $m, \alpha$, and $\beta$ such that

$$
C_{1}\|f\|_{2}^{2} \leq \sum_{k \in \mathbb{Z}}\left|f\left(x_{k}\right)\right|^{2} \leq C_{2}\|f\|_{2}^{2}, \quad \forall f \in V_{m} .
$$

Lemma 3.2. $\inf _{f \in V_{m}} \frac{1}{\|f\|_{2}^{2}} \sum_{k \in \mathbb{Z}}\left|f\left(\frac{m}{2}+k\right)\right|^{2}=0$.

Proof. Since $\varphi_{m}(x)$ is symmetric with respect to $x=\frac{m+1}{2}$ and $\sum_{k \in \mathbb{Z}} \varphi_{m}(x-k)=1$ for any $x \in \mathbb{R}$, it is easy to check that $Z \varphi_{m}\left(\frac{m}{2}, \pi\right)=0$.

For any $\varepsilon>0$, by the continuity of $Z \varphi_{m}(x, \omega)$, there exists some $0<\delta<\pi$ such that

$$
\left|Z \varphi_{m}\left(\frac{m}{2}, \omega\right)\right| \leq \varepsilon, \quad|\omega-\pi| \leq \delta .
$$

Let $C(\omega)=\sum_{k \in \mathbb{Z}} c_{k} e^{-i k \omega}$ be a $2 \pi$-periodic function such that $C(\omega)=1$ for $\omega \in$ $[\pi-\delta, \pi+\delta]$ and $C(\omega)=0$ for $\omega \in[0, \pi-\delta] \cup[\pi+\delta, 2 \pi]$. Define $\hat{f}(\omega)=C(\omega) \hat{\varphi}_{m}(\omega)$. 
Then we have

$$
\begin{aligned}
& \sum_{k \in \mathbb{Z}}\left|f\left(\frac{m}{2}+k\right)\right|^{2}=\frac{1}{2 \pi} \int_{0}^{2 \pi}\left|Z f\left(\frac{m}{2}, \omega\right)\right|^{2} d \omega \\
= & \frac{1}{2 \pi} \int_{0}^{2 \pi}\left|C(\omega) Z \varphi_{m}\left(\frac{m}{2}, \omega\right)\right|^{2} d \omega \leq \frac{\varepsilon^{2}}{2 \pi} \int_{\pi-\delta}^{\pi+\delta}|C(\omega)|^{2} d \omega=\varepsilon^{2} \sum_{k \in \mathbb{Z}}\left|c_{k}\right|^{2} .
\end{aligned}
$$

Since $\left\{\varphi_{m}(\cdot-n): n \in \mathbb{Z}\right\}$ is a Riesz basis for $V_{m}$ with lower bound $A_{m}>0$, the above inequalities show that $\sum_{k \in \mathbb{Z}}\left|f\left(\frac{m}{2}+k\right)\right|^{2}<\frac{\varepsilon^{2}}{A_{m}}$. Since $\varepsilon$ is arbitrary, the conclusion follows.

On the other hand, since $\varphi_{m}(x)$ is compactly supported and absolutely continuous for any $m \geq 1$, we also have

Lemma 3.3. For any $f \in V_{m}, f^{\prime} \in L^{2}(\mathbb{R})$.

We are now ready to give the proof of the main result.

Proof of Theorem 1.1. For any $f \in V_{m}$, let

$$
f_{1}(x)=\frac{f(x)+\overline{f(x)}}{2}, \quad f_{2}(x)=\frac{f(x)-\overline{f(x)}}{2 i} .
$$

Then $f_{1}, f_{2} \in V_{m}$ and $f(x)=f_{1}(x)+i f_{2}(x)$.

Since $f_{p}(x)$ is continuous on $\mathbb{R}$ and $u_{k}(x) \geq 0$, by the first mean value theorem, there are some $x_{p, k} \in\left[x_{k}-\frac{\delta}{2}, x_{k}+\frac{\delta}{2}\right]$ such that

$$
\left\langle f_{p}, u_{k}\right\rangle=f_{p}\left(x_{p, k}\right), \quad p=1,2 .
$$

Put $0<\alpha<\frac{1}{3}(1-\beta-\delta)$. Let

$$
\begin{aligned}
& n_{0}=0, \\
& n_{k}= \begin{cases}\min \left\{n: n>n_{k-1}, x_{n}-x_{n_{k-1}} \geq \alpha\right\}, & k>0, \\
\max \left\{n: n<n_{k+1}, x_{n_{k+1}}-x_{n} \geq \alpha\right\}, & k<0 .\end{cases}
\end{aligned}
$$

Then

$$
\alpha \leq x_{n_{k}}-x_{n_{k-1}} \leq \beta+\alpha, \quad k \in \mathbb{Z} .
$$

For any $k \in \mathbb{Z}$, let $j_{k}$ and $j_{k}^{\prime}$ be the minimum and maximum of $\left\{j: \frac{x_{n_{k-1}}+x_{n_{k}}}{2}<\right.$ $\left.x_{j} \leq \frac{x_{n_{k}}+x_{n_{k+1}}}{2}\right\}$ respectively. Then $\left\{j: j_{k} \leq j \leq j_{k}^{\prime}\right\}_{k \in \mathbb{Z}}$ is a partition of $\mathbb{Z}$.

For any $n_{k}<j \leq j_{k}^{\prime}$, since $j_{k}^{\prime}<n_{k+1}$, by the definition of $n_{k}$,

$$
\min \left\{x_{j}-x_{n_{k}}, x_{n_{k+1}}-x_{j}\right\}<\alpha .
$$

But $x_{n_{k+1}}-x_{j} \geq \frac{x_{n_{k+1}}-x_{n_{k}}}{2} \geq x_{j}-x_{n_{k}}>0$, so $x_{j}-x_{n_{k}}<\alpha$. A similar argument shows that $x_{n_{k}}-x_{j}<\alpha$ for $j_{k} \leq j<n_{k}$. Hence for any $j_{k} \leq j \leq j_{k}^{\prime},\left|x_{j}-x_{n_{k}}\right|<\alpha$. Therefore,

$$
x_{p, j} \in\left[x_{n_{k}}-\alpha-\frac{\delta}{2}, x_{n_{k}}+\alpha+\frac{\delta}{2}\right], \quad j_{k} \leq j \leq j_{k}^{\prime} .
$$


It follows from the continuity of $f_{p}(x)$ and $\sum_{j_{k} \leq j \leq j_{k}^{\prime}}\left(x_{j+1}-x_{j-1}\right)=x_{j_{k}^{\prime}+1}-x_{j_{k}-1}+$ $x_{j_{k}^{\prime}}-x_{j_{k}}$ that there is some $y_{p, k} \in\left[x_{n_{k}}-\alpha-\frac{\delta}{2}, x_{n_{k}}+\alpha+\frac{\delta}{2}\right]$ such that

$$
\begin{aligned}
& \sum_{j_{k} \leq j \leq j_{k}^{\prime}} \frac{x_{j+1}-x_{j-1}}{2}\left|f_{p}\left(x_{p, j}\right)\right|^{2} \\
= & \frac{x_{j_{k}^{\prime}+1}-x_{j_{k}-1}+x_{j_{k}^{\prime}}-x_{j_{k}}}{2} \sum_{j_{k} \leq j \leq j_{k}^{\prime}} \frac{x_{j+1}-x_{j-1}}{x_{j_{k}^{\prime}+1}-x_{j_{k}-1}+x_{j_{k}^{\prime}}-x_{j_{k}}}\left|f_{p}\left(x_{p, j}\right)\right|^{2} \\
= & \frac{x_{j_{k}^{\prime}+1}-x_{j_{k}-1}+x_{j_{k}^{\prime}}-x_{j_{k}}}{2}\left|f_{p}\left(y_{p, k}\right)\right|^{2} .
\end{aligned}
$$

Let $\left\{z_{p, k}: k \in \mathbb{Z}\right\}$ be a rearrangement of $\left\{y_{p, k}: k \in \mathbb{Z}\right\}$ such that $z_{p, k} \leq z_{p, k+1}$. Since $0<x_{n_{k+1}}-x_{n_{k}} \leq \beta+\alpha$ and $\left|y_{p, k}-x_{n_{k}}\right| \leq \alpha+\frac{\delta}{2}$, it is easy to check that every interval with length $\beta+\delta+3 \alpha$ must contain at least one point of $\left\{y_{p, k}: k \in \mathbb{Z}\right\}$. Hence

$$
0 \leq z_{p, k+1}-z_{p, k} \leq \beta+\delta+3 \alpha .
$$

Since $\beta+\delta+3 \alpha<1$, similar to the choice of $x_{n_{k}}$ we can prove that there is a subsequence $\left\{z_{p, i_{k}}: k \in \mathbb{Z}\right\}$ of $\left\{z_{p, k}: k \in \mathbb{Z}\right\}$ such that

$$
\varepsilon \leq z_{p, i_{k+1}}-z_{p, i_{k}} \leq \beta+\delta+3 \alpha+\varepsilon<1
$$

for some $\varepsilon>0$. By Proposition 3.1, there is a positive constant $C_{1}$ depending only on $m, \varepsilon$ and $\beta+\delta+3 \alpha+\varepsilon$ such that

$$
\sum_{k \in \mathbb{Z}}\left|f\left(z_{p, i_{k}}\right)\right|^{2} \geq C_{1}\|f\|_{2}^{2}, \quad \forall f \in V_{m} .
$$

Hence

$$
\sum_{k \in \mathbb{Z}}\left|f_{p}\left(y_{p, k}\right)\right|^{2}=\sum_{k \in \mathbb{Z}}\left|f_{p}\left(z_{p, k}\right)\right|^{2} \geq C_{1}\left\|f_{p}\right\|_{2}^{2}
$$

Noting that

$$
\frac{x_{j_{k}^{\prime}+1}-x_{j_{k}-1}+x_{j_{k}^{\prime}}-x_{j_{k}}}{2} \geq \frac{x_{j_{k}^{\prime}+1}-x_{j_{k}-1}}{2} \geq \frac{x_{n_{k+1}}-x_{n_{k-1}}}{4} \geq \frac{\alpha}{2},
$$

we derive from (3.1) and (3.2) that

$$
\begin{aligned}
(3.3) \sum_{k \in \mathbb{Z}} \frac{x_{k+1}-x_{k-1}}{2}\left|\left\langle f_{p}, u_{k}\right\rangle\right|^{2} & =\sum_{k \in \mathbb{Z}} \frac{x_{k+1}-x_{k-1}}{2}\left|f_{p}\left(x_{p, k}\right)\right|^{2} \\
& =\sum_{k \in \mathbb{Z}} \sum_{j_{k} \leq j \leq j_{k}^{\prime}} \frac{x_{j+1}-x_{j-1}}{2}\left|f_{p}\left(x_{p, j}\right)\right|^{2} \\
& =\sum_{k \in \mathbb{Z}} \frac{x_{j_{k}^{\prime}+1}-x_{j_{k}-1}+x_{j_{k}^{\prime}}-x_{j_{k}}}{2}\left|f_{p}\left(y_{p, k}\right)\right|^{2} \\
& \geq \frac{\alpha C_{1}}{2}\left\|f_{p}\right\|_{2}^{2} .
\end{aligned}
$$

On the other hand, it can be shown (see Section 4) that there is some constant $C_{2}$ such that

$$
\sum_{k \in \mathbb{Z}} \frac{x_{k+1}-x_{k-1}}{2}\left|\left\langle f_{p}, u_{k}\right\rangle\right|^{2}=\sum_{k \in \mathbb{Z}} \frac{x_{k+1}-x_{k-1}}{2}\left|f_{p}\left(x_{p, k}\right)\right|^{2} \leq C_{2}\left\|f_{p}\right\|_{2}^{2} .
$$


Since $f(x)=f_{1}(x)+i f_{2}(x)$ and $f_{1}, f_{2}, u_{k}$ are real functions, we have $\|f\|_{2}^{2}=$ $\left\|f_{1}\right\|_{2}^{2}+\left\|f_{2}\right\|_{2}^{2}$ and $\left|\left\langle f, u_{k}\right\rangle\right|^{2}=\left|\left\langle f_{1}, u_{k}\right\rangle\right|^{2}+\left|\left\langle f_{2}, u_{k}\right\rangle\right|^{2}$. Therefore, (3.3) and (B.4) hold if we substitute $f$ for $f_{p}$. Now the first part of this theorem follows by Lemma 2.2 .

Next we are to prove the second part. We only need to show that the conclusion fails if $\delta=1-\beta$.

First, we consider the case of $\frac{1}{2} \leq \beta<1$.

Let $x_{2 k}=k+\frac{m-1}{2}-\frac{\beta}{2}$ and $x_{2 k+1}=k+\frac{m-1}{2}+\frac{\beta}{2}$. Then $1-\beta \leq x_{k+1}-x_{k} \leq \beta$. Put $\varepsilon_{k}=\frac{1-\beta}{2+|k|}$. Choose some averaging functions $u_{k}(x)$ such that

$$
\begin{aligned}
\operatorname{supp} u_{2 k} & \subset\left[k-1+\frac{m}{2}, k-1+\frac{m}{2}+\varepsilon_{k}\right], \\
\operatorname{supp} u_{2 k+1} & \subset\left[k+\frac{m}{2}-\varepsilon_{k}, k+\frac{m}{2}\right] .
\end{aligned}
$$

Then supp $u_{k} \subset\left[x_{k}-\frac{\delta}{2}, x_{k}+\frac{\delta}{2}\right]$.

For any $\varepsilon>0$, there exists some $f \in V_{m}$ such that

$$
\sum_{k \in \mathbb{Z}}\left|f\left(\frac{m}{2}+k\right)\right|^{2} \leq \varepsilon\|f\|_{2}^{2},
$$

thanks to Lemma 3.2. Since $\varphi_{m}(x)$ is real-valued, we can assume that $f(x)$ is also real-valued. Let $f_{n}(x)=f(x-n), n \geq 1$. Then $f_{n} \in V_{m}$. By the first mean value theorem, there are some $x_{n, 2 k} \in\left[k-1+\frac{m}{2}, k-1+\frac{m}{2}+\varepsilon_{k}\right]$ and $x_{n, 2 k+1} \in$ $\left[k+\frac{m}{2}-\varepsilon_{k}, k+\frac{m}{2}\right]$ such that

$$
\left\langle f_{n}, u_{k}\right\rangle=f_{n}\left(x_{n, k}\right) .
$$

For any $p>0$, we have

$$
\begin{aligned}
\sum_{|k| \geq p}\left|f_{n}\left(x_{n, 2 k}\right)-f_{n}\left(k-1+\frac{m}{2}\right)\right|^{2} \\
\quad=\sum_{|k| \geq p}\left|\int_{0}^{x_{n, 2 k}-k+1-\frac{m}{2}} f_{n}^{\prime}\left(k-1+\frac{m}{2}+t\right) d t\right|^{2} \\
\quad \leq \sum_{|k| \geq p} \varepsilon_{k} \int_{0}^{\varepsilon_{k}}\left|f_{n}^{\prime}\left(k-1+\frac{m}{2}+t\right)\right|^{2} d t \\
\leq \varepsilon_{p} \int_{0}^{\varepsilon_{p}} \sum_{k \in \mathbb{Z}}\left|f_{n}^{\prime}\left(k-1+\frac{m}{2}+t\right)\right|^{2} d t \\
\leq \varepsilon_{p}\left\|f_{n}^{\prime}\right\|_{2}^{2}=\varepsilon_{p}\left\|f^{\prime}\right\|_{2}^{2} .
\end{aligned}
$$

It follows from (3.5) that

$$
\sum_{|k| \geq p}\left|f_{n}\left(x_{n, 2 k}\right)\right|^{2} \leq\left(\varepsilon_{p}^{1 / 2}\left\|f^{\prime}\right\|_{2}+\varepsilon^{1 / 2}\|f\|_{2}\right)^{2}
$$


On the other hand, since

$$
\begin{aligned}
\sum_{|k| \leq n / 2}\left|f_{n}\left(x_{n, 2 k}\right)-f_{n}\left(k-1+\frac{m}{2}\right)\right|^{2} & \leq \sum_{|k| \leq n / 2} \varepsilon_{k} \int_{0}^{\varepsilon_{k}}\left|f_{n}^{\prime}\left(k-1+\frac{m}{2}+t\right)\right|^{2} d t \\
& \leq \int_{0}^{1} \sum_{|k| \leq n / 2}\left|f_{n}^{\prime}\left(k-1+\frac{m}{2}+t\right)\right|^{2} d t \\
& \leq \int_{0}^{1} \sum_{|l| \geq n / 2}\left|f^{\prime}\left(l-1+\frac{m}{2}+t\right)\right|^{2} d t \\
& \leq \int_{|t| \geq \frac{n-m}{2}}\left|f^{\prime}(t)\right|^{2} d t \\
& :=\left\|T_{\frac{n-m}{2}} f^{\prime}\right\|_{2}^{2},
\end{aligned}
$$

we have

$$
\begin{aligned}
\left(\sum_{|k| \leq n / 2}\left|f_{n}\left(x_{n, 2 k}\right)\right|^{2}\right)^{1 / 2} & \leq\left(\sum_{k \in \mathbb{Z}}\left|f_{n}\left(k-1+\frac{m}{2}\right)\right|^{2}\right)^{1 / 2}+\left\|T_{\frac{n-m}{2}} f^{\prime}\right\|_{2} \\
& \leq \varepsilon^{1 / 2}\|f\|_{2}+\left\|T_{\frac{n-m}{2}} f^{\prime}\right\|_{2} .
\end{aligned}
$$

Putting (3.6) and (3.7) together, we have

$$
\begin{aligned}
& \sum_{k \in \mathbb{Z}}\left|f_{n}\left(x_{n, 2 k}\right)\right|^{2}=\sum_{|k|>n / 2}\left|f_{n}\left(x_{n, 2 k}\right)\right|^{2}+\sum_{|k| \leq n / 2}\left|f_{n}\left(x_{n, 2 k}\right)\right|^{2} \\
\leq & \left(\varepsilon_{n / 2}^{1 / 2}\left\|f^{\prime}\right\|_{2}+\varepsilon^{1 / 2}\|f\|_{2}\right)^{2}+\left(\varepsilon^{1 / 2}\|f\|_{2}+\left\|T_{\frac{n-m}{2}} f^{\prime}\right\|_{2}\right)^{2} .
\end{aligned}
$$

A similar argument shows that (3.8) holds if we substitute $f_{n}\left(x_{n, 2 k+1}\right)$ for $f_{n}\left(x_{n, 2 k}\right)$. Therefore,

$$
\sum_{k \in \mathbb{Z}}\left|\left\langle f_{n}, u_{k}\right\rangle\right|^{2} \leq 2\left(\varepsilon_{n / 2}^{1 / 2}\left\|f^{\prime}\right\|_{2}+\varepsilon^{1 / 2}\|f\|_{2}\right)^{2}+2\left(\varepsilon^{1 / 2}\|f\|_{2}+\left\|T_{\frac{n-m}{2}} f^{\prime}\right\|_{2}\right)^{2} .
$$

Noting that $\left\|f_{n}\right\|_{2}=\|f\|_{2}$ for any $n \geq 1$, we have by the above inequality that

$$
\limsup _{n \rightarrow \infty} \frac{1}{\left\|f_{n}\right\|_{2}^{2}} \sum_{k \in \mathbb{Z}}\left|\left\langle f_{n}, u_{k}\right\rangle\right|^{2} \leq 4 \varepsilon
$$

Since $\varepsilon$ is arbitrary, we have $\inf _{f \in V_{m}} \frac{1}{\|f\|_{2}^{2}} \sum_{k \in \mathbb{Z}}\left|\left\langle f, u_{k}\right\rangle\right|^{2}=0$ and hence

$$
\inf _{f \in V_{m}} \frac{1}{\|f\|_{2}^{2}} \sum_{k \in \mathbb{Z}} \frac{x_{k+1}-x_{k-1}}{2}\left|\left\langle f, u_{k}\right\rangle\right|^{2}=0 .
$$

For the general case, suppose that $\frac{1}{r+1} \leq \beta<\frac{1}{r}$ for some integer $r \geq 1$. Let $x_{(r+1) k+l}=k+\frac{m-1}{2}+l \beta-\frac{\beta}{2}, \quad 0 \leq l \leq r$. Then $1-r \beta \leq x_{k+1}-x_{k} \leq \beta$. Define $\varepsilon_{k}=\frac{1-\beta}{2+|k|}$. Choose averaging functions $u_{k}(x)$ such that

$$
\begin{array}{lll}
\operatorname{supp} u_{(r+1) k+l} & \subset\left[k+\frac{m}{2}-\varepsilon_{k}, k+\frac{m}{2}\right], & 1 \leq l \leq \frac{r+1}{2}, \\
\operatorname{supp} u_{(r+1) k+l} & \subset\left[k+\frac{m}{2}, k+\frac{m}{2}+\varepsilon_{k}\right], & \frac{r+1}{2}<l \leq r+1 .
\end{array}
$$


It is easy to check that supp $u_{k} \subset\left[x_{k}-\frac{\delta}{2}, x_{k}+\frac{\delta}{2}\right]$. Similar to the case $\frac{1}{2} \leq \beta<1$ we can prove that

$$
\inf _{f \in V_{m}} \frac{1}{\|f\|_{2}^{2}} \sum_{k \in \mathbb{Z}} \frac{x_{k+1}-x_{k-1}}{2}\left|\left\langle f, u_{k}\right\rangle\right|^{2}=0
$$

If there is a frame $\left\{S_{k}: k \in \mathbb{Z}\right\}$ for $V_{m}$ such that $f=\sum_{k \in \mathbb{Z}}\left(\frac{x_{k+1}-x_{k-1}}{2}\right)^{1 / 2}\left\langle f, u_{k}\right\rangle S_{k}$ holds for any $f \in V_{m}$, by [9. Proposition 3.2.4], we have

$$
\sum_{k \in \mathbb{Z}} \frac{x_{k+1}-x_{k-1}}{2}\left|\left\langle f, u_{k}\right\rangle\right|^{2} \geq \sum_{k \in \mathbb{Z}}\left|\left\langle f, \tilde{S}_{k}\right\rangle\right|^{2} \geq \tilde{A}\|f\|_{2}^{2},
$$

where $\left\{\tilde{S}_{k}(x): k \in \mathbb{Z}\right\}$ is the dual frame of $\left\{S_{k}(x): k \in \mathbb{Z}\right\}$ and $\tilde{A}$ is the lower frame bound. This is a contradiction to (3.9), which completes the proof.

\section{ITERATIVE RECONSTRUCTION ALGORITHM}

By Theorem 1.1, we can reconstruct any $f \in V_{m}$ from local averages by (1.1). However, it may be difficult to find the frame $\left\{S_{k}(x)\right\}$ in practice. In this case, the reconstruction of $f \in V_{m}$ from local averages can be performed by applying the following Feichtinger-Gröchenig algorithm.

Proposition 4.1 (see [13]). Suppose that $\left\{\varphi_{k}: k \in \mathbb{Z}\right\}$ is a frame with bounds $A$ and $B$ for some Hilbert space $\mathcal{H}$. Let $\lambda$ be a constant such that $0<\lambda<\frac{2}{B}$. For any $f \in \mathcal{H}$, define

$$
\begin{aligned}
S f & =\lambda \sum_{k \in \mathbb{Z}}\left\langle f, \varphi_{k}\right\rangle \varphi_{k}, \\
f_{0} & =S f, \\
f_{k+1} & =f_{k}+S\left(f-f_{k}\right), \quad k \geq 0 .
\end{aligned}
$$

Then $\lim _{k \rightarrow \infty} f_{k}=f$ and $\left\|f-f_{k}\right\| \leq \gamma^{k+1}\|f\|$, where $\gamma=\max \{|1-\lambda A|,|1-\lambda B|\}$ $<1$.

For Theorem 1.1, the operator $S$ is of a slightly different form:

$$
S f:=\lambda \sum_{k \in \mathbb{Z}} \frac{x_{k+1}-x_{k-1}}{2}\left\langle f, u_{k}\right\rangle h_{k},
$$

where $h_{k}$ is defined as in Lemma 2.2 .

In the above iterative reconstruction algorithm, the relaxation parameter $\lambda$ plays an important role. If the exact value of the frame bounds are known, then $\lambda=\frac{2}{A+B}$ will be the best one since $\gamma$ will be minimized in this case. Unfortunately, we have only some estimates for the frame bounds in most cases. Whereas it is not difficult to get an estimate for the upper bound of the frame $\left\{\left(\frac{x_{k+1}-x_{k-1}}{2}\right)^{1 / 2} h_{k}: k \in \mathbb{Z}\right\}$ (see below), for the lower bound only existence is known. To ensure the convergence of the iterative reconstruction algorithm, we have to choose a small $\lambda$, which in turn may be responsible for slow convergence. 
To apply Proposition 4.1, an upper frame bound must be found. Let $\left\{x_{k}\right\}, \delta, \beta$, and $\left\{u_{k}\right\}$ be defined as in Theorem 1.1 Define $y_{k}=\frac{x_{k}+x_{k+1}}{2}$. We have

$$
\begin{aligned}
& \left\|f-\sum_{k \in \mathbb{Z}}\left\langle f, u_{k}\right\rangle \chi_{\left[y_{k-1}, y_{k}\right]}\right\|_{2}^{2}=\sum_{k \in \mathbb{Z}} \int_{y_{k-1}}^{y_{k}}\left|f(x)-\left\langle f, u_{k}\right\rangle\right|^{2} d x \\
= & \sum_{k \in \mathbb{Z}} \int_{y_{k-1}}^{y_{k}}\left|\int_{x_{k}-\frac{\delta}{2}}^{x_{k}+\frac{\delta}{2}}[f(x)-f(t)] u_{k}(t) d t\right|^{2} d x \\
\leq & \sum_{k \in \mathbb{Z}} \int_{y_{k-1}}^{y_{k}} \int_{x_{k}-\frac{\delta}{2}}^{x_{k}+\frac{\delta}{2}}|f(x)-f(t)|^{2} u_{k}(t) d t d x \\
= & \sum_{k \in \mathbb{Z}} \int_{y_{k-1}}^{y_{k}} \int_{x_{k}-\frac{\delta}{2}}^{x_{k}+\frac{\delta}{2}}\left|\int_{0}^{t-x} f^{\prime}(x+s) d s\right|^{2} u_{k}(t) d t d x \\
\leq & \sum_{k \in \mathbb{Z}} \int_{y_{k-1}}^{y_{k}} \int_{x_{k}-\frac{\delta}{2}}^{x_{k}+\frac{\delta}{2}} \frac{\delta+\beta}{2} \int_{-\frac{\delta+\beta}{2}}^{\frac{\delta+\beta}{2}}\left|f^{\prime}(x+s)\right|^{2} u_{k}(t) d s d t d x \\
= & \frac{\delta+\beta}{2} \int_{-\frac{\delta+\beta}{2}}^{\frac{\delta+\beta}{2}} \sum_{k \in \mathbb{Z}} \int_{y_{k-1}}^{y_{k}}\left|f^{\prime}(x+s)\right|^{2} d x d s=\frac{(\delta+\beta)^{2}}{2}\left\|f^{\prime}\right\|_{2}^{2} .
\end{aligned}
$$

On the other hand, it can be shown (e.g., see [16]) that $\left\|f^{\prime}\right\|_{2}^{2} \leq \frac{M_{m}}{A_{m}}\|f\|_{2}^{2}$ with

$$
\begin{aligned}
& M_{m}=\sup _{|\omega| \leq \frac{\pi}{2}} \sum_{k \in \mathbb{Z}}|2 \omega+2 k \pi|^{2}\left|\frac{\sin \omega}{\omega+k \pi}\right|^{2 m+2} \leq M_{1}=4, \\
& A_{m}=\inf _{|\omega| \leq \frac{\pi}{2}} \sum_{k \in \mathbb{Z}}\left|\frac{\sin \omega}{\omega+k \pi}\right|^{2 m+2}=\left(\frac{2}{\pi}\right)^{2 m+2} \sum_{k \in \mathbb{Z}} \frac{1}{(2 k+1)^{2 m+2}} .
\end{aligned}
$$

Hence

$$
\sum_{k \in \mathbb{Z}} \frac{x_{k+1}-x_{k-1}}{2}\left|\left\langle f, u_{k}\right\rangle\right|^{2} \leq\left(1+\frac{(\delta+\beta) \sqrt{M_{m}}}{\sqrt{2 A_{m}}}\right)^{2}\|f\|_{2}^{2}:=B\|f\|_{2}^{2}
$$

Therefore, we can choose $0<\lambda<\frac{2}{B}$ to ensure Proposition 4.1 works. However, we cannot give the convergence rate due to the absence of the lower frame bound.

\section{REFERENCES}

1. A.Aldroubi and K.Gröchenig, Beuling-Landau-type theorems for non-uniform sampling in shift invariant spline spaces, J. Fourier Anal. Appl., 6 (2000), 93-103. MR 2001i:42057

2. A.Aldroubi and M.Unser, Families of wavelet transforms in connection with Shannon's sampling theory and the Gabor transform, Wavelets: A tutorial in Theory and Applications (C.K.Chui, Ed.), Academic Press, Boston, 1992, pp.509-528. MR 93a:94003

3. A.Aldroubi and M.Unser, Sampling procedures in function spaces and asymptotic equivalence with Shannon's sampling theory, Numer. Funct. Anal. and Optimiz., 15 (1994), 1-21. MR 95a:94002

4. J.J.Benedetto, Irregular sampling and frames, Wavelets: A Tutorial in Theory and Applications (C.K.Chui, Ed.), Academic Press, Boston, 1992, pp.445-507. MR 93c:42030

5. P.L.Butzer and J.Lei, Errors in truncated sampling series with measured sampled values for non-necessarily bandlimited functions, Functiones et Approximation, 26 (1998), 25-39. MR 99m:42003

6. P.L.Butzer and J.Lei, Approximation of signals using measured sampled values and error analysis, Comm. Appl. Anal., 4 (2000), 245-255. MR 2001i:94033 
7. W.Chen, S.Itoh, and J.Shiki, Irregular sampling theorems for wavelet subspaces, IEEE Trans. Inform. Theory, 44 (1998), 1131-1142. MR 99e:94008

8. C.K.Chui, An Introduction to Wavelets, Academic Press, New York, 1992. MR 93f:42055

9. I.Daubechies, Ten Lectures on Wavelets, SIAM, Philadelphia, 1992. MR 93e:42045

10. H.Feichtinger and K.Gröchenig, Iterative reconstruction of multivariate band-limited functions from irregular sampling values, SIAM J. Math. Anal., 23 (1992), 244-261. MR 93a:94006

11. H.Feichtinger and K.Gröchenig, Irregular sampling theorems and series expansions of bandlimited functions, J. Math. Anal. Appl., 167 (1992), 530-556. MR 93g:41003

12. H.Feichtinger and K.Gröchenig, Error analysis in regular and irregular sampling theory, Appl. Anal., 50 (1992), 167-189. MR 95b:65160

13. H.Feichtinger and K.Gröchenig, Theory and practice of irregular sampling, Wavelets: Mathematics and Applications (J.Benedetto and M.Frazier, Eds.), CRC Press Inc., 1994, pp.305363. MR 94i:94008

14. K.Gröchenig, Reconstruction algorithms in irregular sampling, Math. Comput., 59 (1992), 181-194. MR 93a:41025

15. A.J.E.M.Janssen, The Zak transform and sampling theorem for wavelet subspaces, IEEE Trans. Signal processing, 41 (1993), 3360-3364.

16. Y.Liu, Irregular sampling for spline wavelet subspaces, IEEE Trans. Inform. Theory, 42 (1996), 623-627. MR 97b:94006

17. Y.Liu and G.Walter, Irregular sampling in wavelet subspaces, J. Fourier Anal. Appl., 2 (1995), 181-189. MR 96k:42033

18. W.Sun and X.Zhou, Frames and sampling theorem, Science in China, Series A, 41 (1998), 606-612. MR 99m:94015

19. W.Sun and X.Zhou, Sampling theorem for wavelet subspaces: error estimate and irregular sampling, IEEE Trans. Signal Processing, 48 (2000), 223-226.

20. W.Sun and X.Zhou, Average sampling theorems for shift invariant subspaces, Science in China, Series E, 43 (2000), 524-530. MR 2001m:94030

21. W.Sun and X.Zhou, Average sampling in spline subspaces, Applied Mathematics Letters, 15(2002), 233-237. MR 2003a:94026

22. M.Unser and A.Aldroubi, A general sampling theory for nonideal acquisition devices, IEEE Trans. Signal Processing, 42 (1994), 2915-2925.

23. G.Walter, A sampling theorem for wavelet subspaces, IEEE Trans. Inform. Theory, 38 (1992), 881-884. MR 93e:94006

24. R.G.Wiley, Recovery of band-limited signals from unequally spaced samples, IEEE Trans. Comm., 26 (1978), 135-137.

25. X.G.Xia and Z.Zhang, On sampling theorem, wavelets, and wavelet transforms, IEEE Transactions on Signal Processing, 41 (1993), 2524-2535.

26. R.M.Young, An Introduction to Non-Harmonic Fourier Series, Academic, New York, 1980. MR 81m:42027

27. X.Zhou, W.Sun, On the sampling theorem for wavelet subspaces, J. Fourier Anal. Appl., 5 (1999), 347-354. MR 2001i:42025

Department of Mathematics, Nankai University, Tianjin 300071, People's Republic OF CHINA

E-mail address: sunwch@nankai.edu.cn

Department of Mathematics, Nankai University, Tianjin 300071, People's Republic OF CHINA

E-mail address: xwzhou@nankai.edu.cn 